\title{
Evolución histórica de la higiene corporal: desde la edad antigua a las sociedades modernas actuales
}

\author{
Evolução histórica da higiene pessoal: desde os tempos antigos
}

para as sociedades modernas actuais

\section{Historical evolution of body hygiene: from ancient to modern modern societies}

\begin{abstract}
Francisco José Moreno-Martínez ${ }^{1}$, Carmen Isabel Gómez García², Ana María Hernández-Susarte ${ }^{3}$
${ }^{1}$ Doctor en enfermería por la Universidad de Murcia. Especialista en enfermería pediátrica. Graduado en Fisioterapia. Enfermero del Centro para Personas con Discapacidad El Palmar. Instituto Murciano de Acción Social - IMAS.

${ }^{2}$ Pofesora titular. Doctora en Pedagogía y exdecana de la Facultad de Enfermería de la Universidad de Murcia.

${ }^{3}$ Doctora en Enfermería. Graduada en Fisioterapia y Enfermera en la Residencia de Personas Mayores San Basilio, Instituto Murciano de Acción Social (IMAS).
\end{abstract}

Cómo citar este artículo en edición digital: Moreno-Martínez, F.J., Gómez García,C.I., y Hernández-Susarte, A.Mª (2016).

Evolución histórica de la higiene corporal: desde la edad antigua a las sociedades modernas actuales.

Cultura de los Cuidados (Edición digital), 20( 46). Disponible en: < http://dx.doi.org/10.14198/cuid.2016.46.11>

Correspondencia: Francisco J. Moreno Martínez. Ctra. de Mazarrón, 62. 30120 - El Palmar (Murcia)

Correo Electrónico: frandocfisio@hotmail.com

Recibido: 07/03/2016; Aceptado: 20/08/2016

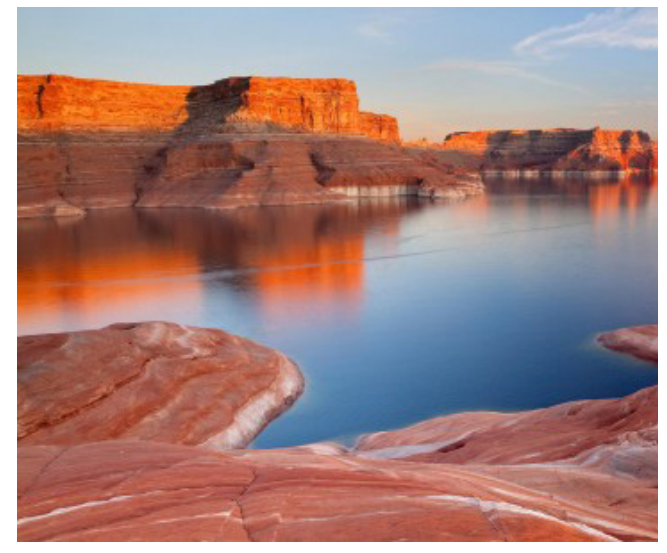

\section{ABSTRACT}

The general aim of inquiry is to study the evolution of corporal hygiene holistically from ancient times to the present. As specific aims we considered: 1-to describe the health consequences of trends in corporal hygiene of modern societies; 2- to describe the role of modern nursing in corporal hygiene care. Methodology and sources: an integrative review was conducted in Medline / Pubmed, Scielo and Lilacs data and a documentation Reverse Search, obtaining a total of 30 jobs. Daily personal hygiene habits have changed considerably throughout history. In ancient Greece and Rome the bathroom acquired social expression and entertainment in addition to therapeutic, and its etymological root is derived from the Greek goddess of health "Hygieia". In medieval times personal hygiene deserved only an aesthetic importance linking the disease with pobredumbre air, as in the Renaissance where the phantasmagoria around the bathroom also arises. In the nineteenth century, and advances in microbiology, the hygienist movement emerged also enriching hygiene as a concept. Currently developed societies achieve high levels of personal hygiene which recent research suggests that may be counterproductive to health, reconsidering what the best personal hygiene habits based on scientific evidence.

Keywords: Corporal Hygiene; Cultural Evolution; Nursing Care; Cultural History. 


\section{RESUMO}

O objetivo geral da pesquisa é estudar a evolução da higiene pessoal de forma holística desde os tempos antigos até o presente. Como objetivos específicos podemos definir: 1-descrever as consequências para a saúde de tendências da higiene pessoal das sociedades modernas; 2 - descrever o papel da enfermagem moderna no cuidado de higiene corporal. Metodologia e as fontes: uma revisão integrativa em bases de dados Medline/ Pubmed, Scielo e Lilacs foi realizada, e uma pesquisa inversa de documentação, obtendo um total de 30 postos de trabalho.Diários hábitos de higiene pessoal mudaram consideravelmente ao longo da história. Na Grécia e Roma antigas banheiro adquiriu expressão social e de entretenimento, além de terapêutica, e sua raiz etimológica é derivado da deusa grega da saúde "Hygieia”. Nos tempos medievais higiene pessoal merecia única uma importância estética que liga a doença com ar pobredumbre, como no Renascimento, onde a fantasmagoria em torno do banheiro também surge. No século XIX, e avanços em microbiologia, o movimento higienista surge também o enriquecimento de higiene como um conceito. Sociedades desenvolvidas atualmente alcançar altos níveis de higiene pessoal que pesquisas recentes sugerem que pode ser contraproducente para a saúde, reconsiderando o que os melhores hábitos de higiene pessoal, baseada em evidências científicas.

Palavras-chave: Higiene Corporal; Evolução Cultural; Cuidados de Enfermagem; História Cultural.

\section{RESUMEN}

El objetivo general del estudio es estudiar la evolución de la higiene corporal de forma holística desde la edad antigua a la actualidad. Como objetivos específicos nos planteamos: 1-describir las consecuencias para la salud de las tendencias en higiene corporal de las sociedades modernas; 2- describir el rol de la enfermería moderna en los cuidados higiénicos corporales. Metodología y fuentes: se realizó una revisión integradora en las bases de datos Medline/Pubmed, Scielo, y Lilacs y búsqueda inversa de documentación, obteniendo un total de 30 trabajos. Los hábitos cotidianos de higiene corporal han sufrido importantes variaciones a lo largo de la historia. En la antigua Grecia y Roma el baño adquiría expresión social y de ocio además de terapéutica, incluso su raíz etimológica deriva de la Diosa griega de la salud "Hygieia". En la época medieval la higiene corporal sólo merecía una importancia estética relacionando la enfermedad con la pobredumbre del aire, al igual que en el renacimiento donde además surge la fantasmagoría en torno al baño. Con el siglo XIX y los avances en microbiología surge el movimiento higienista enriqueciendo además a la higiene como concepto. Actualmente las sociedades desarrolladas alcanzan elevados niveles de higiene corporal donde recientes investigaciones señalan que pueden ser contraproducentes para la salud, replanteándose cuáles son los mejores hábitos de higiene corporal basados en la evidencia científica.

Palabras clave: Higiene Corporal; Evolución Cultural; Atención de Enfermería; Historia Cultural.

\section{INTRODUCCIÓN}

La importancia conferida a la higiene corporal por el ser humano a lo largo de su historia ha sido fluctuante: teniendo consideración de actividad comunitaria en la edad antigua, pasando por el recelo al agua de la edad media, ser un lujo poco frecuente hasta hace menos 
de un siglo. Actualmente, aunque es aceptada por las sociedades modernas como una rutina diaria privada o individual (Hand, Shove y Southerton, 2005), diversos estudios se plantean las consecuencias negativas para la salud de estos nuevos hábitos adquiridos denominados de sobrehigienización (Logan, Katzman y Balanzá-Martínez, 2015) fundamentados en la hipótesis de la higiene de Strachan (1989) y en las nuevas técnicas analíticas para el estudio del microbioma corporal.

La higiene corporal actualmente es considerada una necesidad básica, teñida de componentes culturales (Gerez Alum, 2008, p. 380), dirigida a asegurar y mantener la salud del individuo (Araque y Colmenar, 2011, p. 517), del grupo doméstico y de la colectividad, pero para poder comprender el significado que hoy se concede a la palabra higiene en nuestra cultura, es importante conocer la evolución de dicho término y la interpretación que le ha sido asignada a lo largo de la historia hasta nuestros días.

El concepto de higiene corporal puede derivar en múltiples interpretaciones siendo quizás más adecuado referirnos a culturas de la higiene, en plural, aunque existen estudios que demuestran que la higiene "más allá del significado que cada grupo social puede haberle dado a esa cultura, la higiene individual y colectiva han devenido en prácticas civilizatorias y de socialización, (...) siendo interiorizadas (...) no necesaria o exclusivamente, como resultado de una suerte de resignada aceptación, sino como una evidencia de las ventajas y mejoras que podrían lograr" (Márquez, Casas y Estrada, 2004, p.33). Los logros que una cultura desarrolla en el campo de la higiene, están influidos directamente por los conocimientos científicos y tecnológicos que esa determinada cultura posea en un determinado momento (Sigerist, 1987, en García Ballester y Mc Vaugh, 1996, p.482), por tanto, el estudio de la evolución histórica de la higiene corporal nos permite comprender las motivaciones de los hábitos higiénicos cotidianos empleados en las principales etapas de la historia de la humanidad.

El presente artículo pretende realizar una detallada descripción de la evolución histórica de la higiene corporal referida tanto al propio concepto como a los hábitos higiénicos y su relación con las tendencias sociales y conocimientos sanitarios de la época, iniciando el estudio en la edad antigua hasta alcanzar las tendencias actuales de las sociedades modernas y las principales e incipientes líneas de investigación abiertas en torno a este tema. Finalmente se analiza el rol que ha desempeñado la enfermería respecto a la higiene corporal desde su concepción moderna como profesión, hasta el papel que desarrolla en la actualidad en la que es considerada una necesidad básica para el ser humano.

El objetivo general de la presente investigación es estudiar la evolución de la higiene corporal de forma holística desde la edad antigua a la actualidad. Como objetivos específicos nos planteamos: 1-describir las consecuencias para la salud de las tendencias en higiene corporal de las sociedades modernas; 2- describir el rol de la enfermería moderna en los cuidados higiénicos corporales.

\section{METODOLOGÍA Y FUENTES}

Se realizó una revisión integradora de la literatura con las siguientes etapas: identificación de la hipótesis, objetivos de la revisión y establecimiento de criterios de inclusión y exclusión de artículos; etapa de búsqueda exhaustiva de literatura; evaluación de la calidad de las fuentes primarias; análisis de los resulta- 
dos; y presentación de los principales resultados (Whittemore y Knafl, 2005).

Las preguntas orientadoras de la investigación fueron: ¿cómo han evolucionado los hábitos higiénicos corporales desde la edad antigua hasta la actualidad?; ¿cómo ha evolucionado etimológicamente la higiene corporal?; ¿cuál es el papel que ha desempeñado la enfermería como profesión moderna?; ¿cuál es la tendencia actual en higiene corporal de las sociedades modernas y cómo afecta a su salud?.

La selección de la literatura de efectuó durante el mes de septiembre de 2015 utilizando las bases de datos: Medline/Pubmed, Scielo, y Lilacs. Para las bases de datos en inglés se emplearon los $<<$ Major MesH $>>$ : $<<$ Hygiene $>>$; $<<$ Renaissance $>>$; $<<$ Old World $>>$; $<<\mathrm{Me}$ dieval History $>>$; $<<$ Human Microbioma $>>$; $<<$ History Nursing $>>$ y el $<<$ Minor MesH $>>$ : $<<$ Hygiene Hypothesis $>>$. Para las bases de datos en castellano se utilizan los DeCS: $<<\mathrm{Hi}-$ giene $>>$; $<<$ Historia Medieval $>>$; $<<$ Historia Antigua $>>$; $<<$ Historia del siglo XV [hasta el $\mathrm{XXI}]>>$; $<<$ Hipótesis de la Higiene $>>$; $<<$ Historia de la Enfermería $>>$; y el término en lenguaje natural $<<$ Microbioma Humano $>>$. Las pesquisas se completaron con una búsqueda inversa de artículos para acceder a las fuentes primarias, utilizándose para los libros la herramienta de búsqueda "Google libros".

Entre los criterios de inclusión se encuentran: idioma inglés y castellano, estar disponible electrónicamente, tener acceso al documento de forma completa, y guardar relación directa con alguno de los objetivos planteados.

Se seleccionaron los documentos considerados de interés, en primer lugar mediante lectura del título y resumen. En segundo lugar se procedió a la lectura del texto completo de los artículos, extrayendo los resultados que respondían a los objetivos del estudio. En ter- cer término, la discusión de los resultados. En cuarto lugar, la presentación de los resultados, y finalmente, la presentación y divulgación de la revisión. En primer término, la búsqueda protocolizada en las bases de datos obtiene un total de 1182 artículos. Tras la lectura de los resúmenes y cumpliendo los criterios de inclusión, y atendiendo las preguntas orientadoras de la investigación, fueron seleccionados 17 artículos para su análisis cuya búsqueda inversa de literatura permitió seleccionar otros 13 documentos, obteniendo un total de 30 trabajos.

\section{DESARROLLO DEL TEMA}

\section{La higiene en la antigua Grecia y el Imperio romano (importancia higiénica de los baños)}

La palabra higiene está estrechamente relacionada con el concepto de salud y con la antigua Grecia desde su propia raíz etimológica, ya que procede del griego hygiés, sano. Designa el arte y la ciencia de la conservación de la salud. Su raíz también procede del nombre "Hygieia", nombre que los griegos dieron a la diosa griega de la salud, hija del dios Asclepio (Esculapio para los romanos) quién, según la mitología griega, enseñó la medicina a los hombres (Araque y Colmenar, 2011; Criado, 2004a; Sánchez González, 2002).

Las civilizaciones antiguas ya conocían las propiedades higiénicas y terapéuticas del baño, formando además parte de sentimientos religiosos y rituales propios de cada época (Azcona, 2006). Ya existían los baños públicos en Grecia cerca de 1000 años antes de Jesucristo, siguiendo el ejemplo de los orientales a quiénes se les considera los primeros en construir edificios públicos a tal fin (De Paula, 1846). La medicina griega prestó atención sobre todo a la higiene personal, basada en la teoría humoral, intentando alcanzar un equi- 


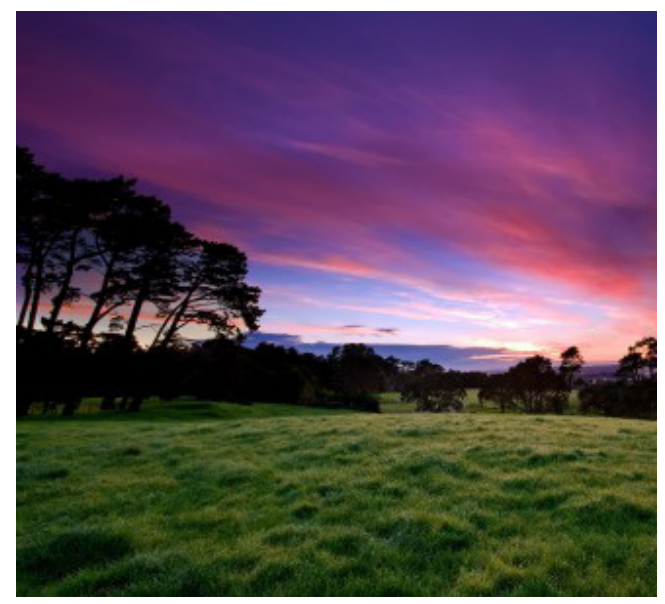

librio entre los cuatro humores principales: la sangre, la flema, la bilis amarilla y la bilis negra (Sánchez González, 2002; Volcy, 2004). Una de las terapéuticas provenientes de la medicina hipocrática para restablecer el equilibrio humoral eran los baños, puesto que "el agua traía efectos benéficos, además de purificar el alma por la inmersión que lava y renueva" (Volcy, 2004, p. 81).

Los romanos, siempre admiradores de los griegos, adoptaron el baño como algo habitual, pasando en ellos buena parte de la jornada. Según De Paula, (1846, p. 305) "Pompeyo mandó construir los primeros hacia el año 65 antes de Jesucristo", pero es a partir de la época del Imperio cuando los baños públicos comienzan a multiplicarse en Roma. Como comenta Blázquez Martínez (2000): "Las termas públicas desempeñaban un importante papel social, además de la función primordial de la higiene". Espinosa y López (1997, p.259) igualmente, afirman que: "Los baños que se construyen en ámbitos urbanos se asocian a fines de higiene y ocio". En esta línea, Molina Villar (2004, p. 40) afirma que los baños "se integraban en el modo de vida del mundo romano de la época imperial, donde el agua, la higiene y el baño, estaban muy presentes".
Roma introdujo los baños en Hispania al final de la Guerra Numantina (año 133 a.C.) y se va extendiendo y asimilando por las distintas comunidades peninsulares. Las ciudades tenían muchas fuentes públicas. La red de cloacas estaba muy perfeccionada. En algunas ciudades modernas siguen en uso o se superpusieron a ellas la red de alcantarillado actual. Roma cuidó siempre los sistemas de higiene con el fin de evitar enfermedades, ello incluía la instalación de letrinas en los grandes edificios públicos (Blázquez Martínez, 2000).

\section{La higiene desde la Edad Media al siglo XVIII}

En la Edad Media, algunos historiadores ven en la falta de higiene de las ciudades medievales no solamente una cuestión material o de grado, cuantitativa, sino una cuestión cualitativa; la percepción social de la suciedad era distinta por ejemplo a la visión higiénicomédica de mediados de siglo XX o a la ecológica de finales del mismo siglo (Fossier, 1996). Asimismo, el antiguo menosprecio renacentista hacia esa época, relacionó al hombre medieval con la barbarie, la suciedad, la ingenuidad infantiloide que le llevaría a menospreciar las consecuencias de la falta de higiene respecto de los contagios. No obstante, la limpieza en aquella época, era vista como un requisito necesario para la salud; evidentemente no porque fuese caldo de cultivo bacteriológico (lo que supondría un flagrante anacronismo), sino porque viciaba el aire, lo corrompía.

Jaume d'Agramunt, en su Règim de Pestilència, de 1348, se refería al vínculo causal o coadyuvante de la suciedad respecto a la generación de la peste. Sus conocimientos médicos, basados en el galenismo aristotélico, le llevaban a creer que la enfermedad era fruto de una mutación del entorno, una podredumbre del aire. En ningún momento de su obra 
llega a referirse a la suciedad del propio individuo porque ésta no modificaba su entorno. Ciudades que adoptaron medidas preventivas relacionadas con la higiene vieron cómo sus estragos remitieron considerablemente, como es el caso de Milán y Nuremberg (Gerez Alum, 2008), o Sevilla (Córdoba, 1998, p. 283).

Piédrola (2002, pp. 7-8) señala que en la Edad Media, "el desprecio de lo mundano y el cambio en las costumbres de la higiene personal, por considerar inmoral la contemplación del propio cuerpo lograron que se abandonase el baño corporal". La limpieza personal en la Edad Media, tenía como objetivo la decencia antes que la higiene. Antes que la salud, lo que domina a llevar a cabo estas prácticas es la apariencia, de ahí a que principalmente se asearan las manos y la cara. Esta ideología continúa en el siglo XVI y XVII donde la apariencia de limpieza personal se extrapola también a la vestimenta, y al perfume que tenía un papel purificante del aire (Vigarello, 1991).

En los siglos XVI y XVII surge la fantasmagoría en torno al agua, siendo percibida, según informa Vigarello (1991, p. 15), como “algo capaz de infiltrarse en el cuerpo, por lo que el baño, en el mismo momento, adquiere un estatuto muy específico. Parece que el agua caliente, en particular, fragiliza los órganos, dejando abiertos los poros a los aires malsanos". Los baños públicos se prohíben progresivamente y van desapareciendo del paisaje urbano. Como ejemplo, en París, de los treinta baños registrados en el siglo XIV, sólo queda alguno a finales del siglo XVII (Vigarello, 2006, p. 66). El rechazo del agua no suprime la práctica de la limpieza, de modo que prolifera la limpieza en seco. "Esta amplísima desaparición puede hacernos pensar en un retroceso en las normas higiénicas" en esta época (Vigarello, 1991, p. 35).
Los tratados de higiene del siglo XVIII racionalizan los poros de la piel como facilitadores de salida a las transpiraciones. "La Limpieza que en el siglo XVII sólo era la traducción de razones estéticas y de civilidad se dirige ahora con mayor claridad hacia las funcionalidades" (Vigarello, 1991, p. 180). Sin embargo esto no implica una revolución inmediata de las prácticas de la ablución. El cuidado de la piel estará subordinado al de la ropa todavía durante mucho tiempo. "Lo que nace a partir de los años 1760 es, por lo menos, la posibilidad de una limpieza totalmente nueva" (Vigarello, 1991, p. 180). Algunos autores del momento como un tratado de higiene escrito por Buchan (1769, en Sánchez González, 2002, p. 246) instan a la atención del gobierno respecto a la higiene corporal, citando que "la limpieza debe ser objeto de atención pública. No es suficiente que yo me limpie, mientras que mi vecino por falta de limpieza afecta a mi salud al mismo tiempo que la suya”.

\section{Movimiento higienista (siglo XIX) y princi- pios del siglo $\mathrm{XX}$}

"Hasta el siglo XIX puede decirse que Europa era un continente sucio en comparación con el resto del mundo" (Sánchez González, 2002, p. 246). El término higiene tiene su más amplio desarrollo fundamentalmente en este siglo, apareciendo el llamado movimiento higienista a mediados del siglo XIX constituyendo un movimiento internacional con diversos orígenes y ramificaciones en los ámbitos médico-sanitarios y sociales. La microbiología pausteriana transforma desde 1870-1880 la percepción de la limpieza. David (1897, en Vigarello, 1991, p. 253) afirma que "la limpieza es la base de la higiene, puesto que consiste en alejar de nosotros toda suciedad y, por ello, todo microbio". 
Moreno y Viñao (2000, p. 20) nos dan a entender la importancia del movimiento higienista "a partir de presupuestos higiénicos, pretende regular la vida de los individuos, de las familias y de los grupos sociales en toda su amplitud, siendo la escuela y la familia los que constituyeran, desde su inicio, dos de los lugares privilegiados de intervención del higienismo". La familia ya era señalada como el mejor manantial de educación en higiene, indicando Tolosa Latour (1900, p. 24, en Campos, 1998, p. 346) que la ignorancia de los principios de higiene en el hogar, es causa degenerativa de los niños, dejando el papel de educadoras a las mujeres en su rol social de madres (Campos, 1998, p. 346).

El higienismo se formulaba como movimiento científico, "acercándose al estudio de las condiciones y factores ambientales, que influían de manera negativa en la salud humana (...). El objetivo principal del higienismo era el conservar la salud y prolongar la vida” (Molina Villar, 2004, p. 101).

La higiene corporal, y la limpieza se fueron interiorizando. "Proliferaron en los dominios privados en este siglo los baños, los lavabos. (...) La limpieza personal se convirtió en un signo de educación y gentiliza moral" (Sánchez González, 2002, p. 246). El baño se irá incorporando "como práctica habitual en relación directa con el estado de salud (...). Las personas que habitualmente usan los baños, están menos expuestas a las enfermedades" (Molina Villar, 2004, p. 273).

En la definición de higiene coincidían básicamente todos los autores, como el arte de mantener la salud en su integridad, conservarla en tal estado, prevenir la enfermedad, y producir longevidad o alargar la vida. Algunos como García Gómez (1920, en Araque y Colmenar, 2011, p. 1) llegaban a considerar que "su conocimiento debe generalizarse como el de la física, la química y las matemáticas, pertenece a las masas como la religión y la moral". La higiene formó parte de los planes de estudio españoles de esta época, recogiéndose en los mismos por primera vez en el Reglamento General de Instrucción Pública de 1821 e implantándose de forma real en los centros educativos en 1868 donde permaneció, al menos en los planes de estudios de mayor vigencia, hasta 1936 (Araque y Colmenar, 2011). En 1900, higiene y educación constituían una pareja inseparable. "En España, desde 1901 la enseñanza de la higiene y la fisiología, con fines preventivos, se declaraba obligatoria en todas las escuelas, constituyendo una asignatura obligatoria en todos los programas escolares" (Criado Rodríguez, 2004b, p. 1).

\section{Conceptualización moderna del término hi- giene}

Según la última edición del Diccionario de la lengua española (Real Academia Española, 2014), se denomina higiene: 1. "parte de la medicina que tiene por objeto la conservación de la salud y la prevención de enfermedades"; 2. "Limpieza o aseo". El Diccionario de la lengua española (Real Academia Española, 2014) considera la higiene privada como aquella "de cuya aplicación cuida el individuo", y la higiene pública la que "en cuya aplicación interviene la autoridad, prescribiendo reglas preventivas".

Por definición, como afirma De Freminville (2007, p. 90), "la higiene es una ciencia que enseña las medidas adecuadas que se han de tomar para conservar la salud", lo que implica no únicamente la limpieza o aseo personal sino "tocar todas las vertientes de la vida de una persona" como la alimentación, la prevención y los cuidados médicos, la actividad física e intelectual, las relaciones sociales, la cultura, el trabajo, la limpieza y cuidados personales. 
Aunque la definición científica y oficial actual del término higiene sigue manteniendo la conceptualización heredada del movimiento higienista, el uso coloquial del término higiene generalmente es referido a la limpieza, y especialmente a cualquier práctica enfocada a la ausencia o reducción de los perjudiciales agentes infecciosos (Jumaa, 2005, p. 4). Es por esta razón que la higiene es considerada un término que ha sido deslustrado en nuestros tiempos (Criado-Rodríguez, 2004a, p. 1).

\section{Cultura contemporánea de la sobrehigieniza-} ción corporal: factores determinantes y consecuencias para la salud

La sociedad actual asume la higiene corporal como parte de su cotidianeidad, un valor que forma parte de su cultura. Pero las prácticas y hábitos cotidianos que hemos adoptado en higiene corporal están influenciados por diversos factores, responsables de la evolución de nuestras rutinas.

La mayoría de los autores recomiendan una higiene corporal mediante el baño o ducha con una periodicidad que debería ser diaria. Esto conlleva a que una frecuencia inferior de aseo corporal sea considerada social y físicamente incómoda. Sin embargo, como hemos señalado en la revisión histórica de la conceptualización de la higiene, esto no siempre ha sido así.

Hand et al. (2005) fundamentalmente señalan tres factores como posibles responsables de las tendencias actuales de higiene corporal individual (referente al aseo corporal a través de la ducha): el desarrollo tecnológico de las infraestructuras de agua, electricidad y las innovaciones asociadas al baño; la intervención del estado y del gobierno en el establecimiento de la hegemonía social y cultural de los beneficios de la higiene corporal para la salud; y la organización temporal de la vida diaria. Estos

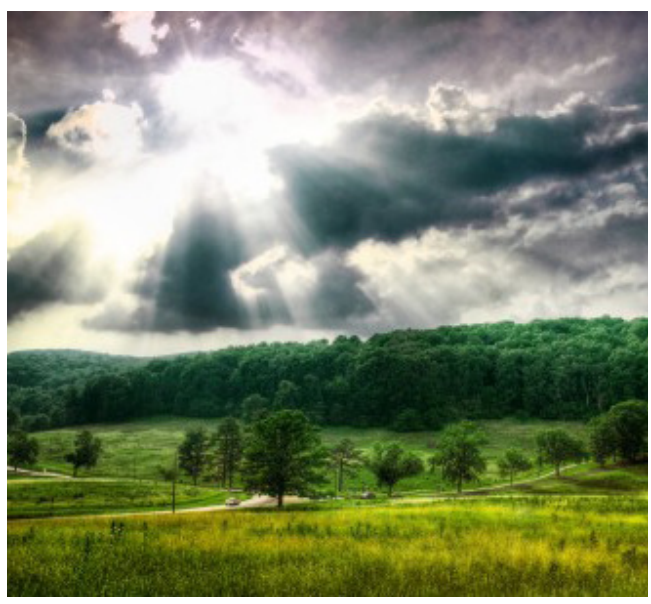

autores también señalan que ha podido influir en nuestros hábitos higiénicos "las estrategias de las empresas implicadas en la comercialización y la mercantilización de los conceptos de bienestar personal y la atención a través de la segmentación continua".

Las consecuencias para la salud de la falta de contacto con los "normalmente inocuos microbios del medio ambiente" fueron inicialmente expuestas por René Dubos en 1973 afirmando que podían comprometer el normal desarrollo del sistema inmunológico, reconociendo que sus sugerencias no estaban en línea con el pensamiento contemporáneo (Logan, Katzman y Balanzá-Martínez, 2015). Posteriormente el científico británico Strachan en 1989 planteó la "hipótesis de la higiene" la cual sugiere que la reducción en la exposición a microorganismos, fundamentalmente en la infancia (Kramer et al., 2013), debido a la mayor higiene personal y la mejora de las condiciones de salud de los países desarrollados, contribuye al aumento de la incidencia de los trastornos autoinmunes como la esclerosis múltiple (Pehlivan et al., 2011), enfermedades alérgicas, enfermedad inflamatoria intestinal (Kramer et al., 2013) o el alzhéimer (Fox, Knapp, Andrews y Fincher, 2013). 
Sin embargo, en base a estos resultados, no debe concluirse que se puede prescindir con seguridad de las normas de higiene, pues, como afirma la OMS, la estrategia preventiva de la higiene ha sido la responsable de la duplicación de la esperanza de vida desde 1870, por lo que las reglas sociales de la higiene como el lavado de manos después de ir al baño, deben mantenerse (Kramer et al., 2013).

\section{Influencia de la higiene en el equilibrio del microbioma corporal}

El estudio de la microbiología de la piel humana se inició hace décadas, siendo el científico Joshua Lederberg en 2001 quien acuñó el término "microbioma" definido como la comunidad ecológica de microorganismos comensales, simbióticos y patógenos que literalmente comparten nuestro espacio corporal" (Lederberg, 2001), y que tienden a mantener un estado de equilibrio, resistiendo cambios bruscos en la estructura de la comunidad. Este proceso puede proteger al huésped de patógenos microbianos excluyéndolos de cada ecosistema (Fredricks, 2001, p. 167). Se ha observado que el microbioma corporal influye en la modulación del sistema inmune y metabólico, y es esencial para el completo desarrollo funcional de las células T (linfocitos T), modulando su expansión y activación, evitando enfermedades infecciosas y autoinmunes (Grice y Segre, 2011).

Diversos factores, tanto externos como propios del huésped, pueden alterar el ecosistema de la piel, entre ellos: la higiene y el uso de jabones y cosméticos. "Actividades que parecen tan triviales para nosotros, como tomar una ducha, pueden ser el equivalente a un huracán para los microbios que habitan en la piel, con cambios en el paisaje y la estructura de la población” (Fredricks, 2001, p. 167). En esta línea, los estudios de cartografía humana en 3D de Bouslimani et al. (2015) revelan que la composición molecular de la piel está influida por nuestras rutinas diarias, incluidas la aplicación de productos de higiene, sirviendo sus investigaciones como base para el estudio de las relaciones de la piel humana con la higiene, la microbiota y el medio ambiente.

\section{La enfermería como promotora del cuidado en higiene corporal}

La relación entre la enfermería y la higiene tiene un largo recorrido histórico y está y ha estado presente en múltiples corrientes teóricas.

Podemos establecer la relación entre higiene corporal y enfermería desde la concepción de la misma como profesión, a mediados del siglo XIX, siendo Florence Nightingale, teórica considerada fundadora de la enfermería moderna, quien establece las primeras diferenciaciones entre esta disciplina incipiente, y el trabajo de los asistentes domésticos y las tradicionales “enfermeras". Nightingale enfatizó las cuestiones relacionadas con la higiene personal, además de su constante estudio sobre correlaciones entre las variables higiene, pobreza y salud (Amaro, 2004). En su obra más divulgada "Notas de enfermería: ¿qué es y qué no es?" (1859), incide en que el proceso de recuperación de la persona enferma está entorpecido por la necesidad de aire fresco, o de luz, o de calor, o de tranquilidad, o de limpieza, o de puntualidad y cuidado en la administración de la dieta" (Nightingale, 1990, p. 2).

Desde entonces la atención a la higiene corporal es considerada una parte esencial vinculada a los cuidados de enfermería. Virginia Henderson, teórica enfermera, describe en 1966, en su libro “The Nature of Nursing", uno de los modelos conceptuales más importantes 
de la enfermería moderna, contemplando la higiene corporal dentro de las 14 necesidades básicas que la persona debe satisfacer para mantener su integridad y promover su desarrollo y crecimiento. Dicha necesidad queda enunciada como: "Mantener la higiene corporal y la integridad de la piel".

Dorothea E. Orem teórica enfermera es posible que se inspirara en V. Henderson para el desarrollo de su modelo, compuesto por tres teorías: del autocuidado; del déficit de autocuidado; y de sistemas de enfermería. La higiene corporal queda representada en los requisitos universales de la teoría del autocuidado, definidos como "los comportamientos que por lo general se consideran dentro de los límites normales para los seres humanos. Constituyen los objetivos requeridos que deben alcanzarse a través del autocuidado para el mantenimiento de la integridad estructural y funcional en las diversas etapas del ciclo vital" (Vega y González, 2007, p. 31).

Actualmente la OMS define a la enfermería como una profesión que "abarca la atención autónoma y en colaboración dispensada a personas de todas las edades, familias, grupos y comunidades, enfermos o no, y en todas circunstancias. Comprende la promoción de la salud, la prevención de enfermedades y la atención dispensada a enfermos, discapacitados y personas en situación terminal". De la definición de enfermería de la OMS interpretamos como la higiene corporal debe ser atendida por la profesión enfermera, inmersa en la "atención autónoma" de la disciplina, como medio para la promoción de la salud y prevención de la enfermedad en la población sana, o como ayuda en el restablecimiento de la salud en enfermos. Para ello los profesionales de enfermería no sólo cuentan con la aplicación de cuidados en higiene corporal cuan- do "el individuo experimenta un deterioro de la habilidad para llevar a cabo o completar por sí mismo las actividades de baño/higiene personal" (diagnóstico del déficit de autocuidado: baño/higiene), sino que son los profesionales responsables de establecer programas de educación para la salud en higiene corporal entre la población sana.

\section{CONSIDERACIONES FINALES}

La higiene corporal siempre ha formado parte de la historia de la humanidad, y aunque ha pasado por épocas donde su importancia para la salud ha podido ser menospreciada, nunca ha dejado de aplicarse de un modo u otro. Actualmente las sociedades modernas disponen de mayores facilidades para la obtención de medios que facilitan la higiene corporal, existiendo investigaciones que advierten de los riesgos para la salud de una higiene excesiva, sobre todo en la infancia. Aunque no se debe prescindir de las prácticas de higiene corporal como principal medio de prevención de enfermedades infecciosas, se debe fomentar la investigación dirigida al estudio de aquellos hábitos de higiene corporal más adecuados para la prevención enfermedades y conservar las mejores condiciones de salud en las que la higiene corporal en cualquiera de sus dimensiones (bucodental, cabello, piel corporal, ótica, podal, íntima, manos...) puede ser responsable. Aún existen muchas controversias y conocimiento por generar sobre las mejores prácticas higiénicas corporales cotidianas. La investigación en esta materia por parte de enfermería debe fomentarse y ser notoria, al tratarse de un aspecto básico para los cuidados del ser humano que afecta de forma directa a esta disciplina sanitaria. Además, la enfermería, profesión sanitaria de importante acceso a la población, debe incluir la transmisión de 
conocimientos y hábitos basados en la evidencia científica, sobre todo en la infancia donde es más fácil modificar y generar conductas que perdurarán en la vida adulta.

\section{BIBLIOGRAFÍA}

- Amaro Cano, M.C. (2004). Florence Nightingale, la primera gran teórica de enfermería. Revista cubana de enfermería, 20(3). Recuperado de http://scielo. sld.cu/scielo.php?script=sci_arttextypid=S086403192004000300009

- Araque Hontangas, N., y Colmenar Orzaes, C. (2011). Salud y educación. Reflexiones en torno a la higiene en los textos para la enseñanza secundaria. ARBOR Ciencia, Pensamiento y Cutura, 187(749), 513-524.

- Azcona, L. (2006). Higiene corporal. Farmacia Profesional, 20(4), 66-69.

- Blázquez Martínez, J.M. (2000) Los pueblos de España y el Mediterráneo en la antigüedad. Estudios de arqueología, historia y arte. Madrid: Cátedra

- Bouslimani, A., Porto, C., Rath, C.M., Wang, M., Guo, Y., González, A., Dorrestein, P.C. (2015). Molecular cartography of the human skin surface in 3D. Proceedings of the National Academy of Sciences, 112(17), 2120-2129.

- Campos Marín, R. (1998). La teoría de la degeneración y la medicina social en España en el cambio de siglo. Llull, 21, 333-356.

- Córdoba de la Llave, R. (1998). La vida cotidiana en la España Medieval. En M.A. García Guinea (Ed.), Higiene urbana y doméstica en las poblaciones castellanas del XV (pp.281-302). Madrid: Polifemo.

- Criado-Rodríguez, E. (2004a). Reflexiones Coloquiales Sobre La Vieja Higiene y La Moderna Salud Pública I (Aplicadas al Origen de la Educación Para La Salud). A Tu Salud, 45, 1.

- Criado Rodríguez, E. (2004b). Reflexiones Coloquiales Sobre La Vieja Higiene y La Moderna Salud Pública II (Aplicadas al Origen de la Educación Para La Salud). A Tu Salud, 46, 1.

- De Freminville, B. (2007). La higiene en la vida cotidiana.
Revista Síndrome de Down, 24, 90-99.

- De Paula Mellado, F. (1846). Diccionario Universal de Historia y de Geografía. Madrid: Autor.

- Espinosa Ruíz, U. y López Domech, R. (1997). Agua y cultura antigua en el Alto-Medio Ebro. En M.J. Pérex Agorreta (ed.), Termalismo antiguo. Actas del I Congreso Peninsular (pp.259265). Arnedillo (La Rioja), 3-5 octubre 1996 (pp.259-265). Madrid: Universidad Nacional de Educación a Distancia.

- Fredricks, D.N. (2001). Microbial ecology af human skin in health and disease. JID Symposium Proceedings, 6(3), 167-169.

- Fossier, R. (1996). La Sociedad Medival. Barcelona: Crítica

- Fox, M., Knapp, L.A., Andrews, P.W. y Fincher, C.L. (2013), Hygiene and world distribution of alzheimer's disease. Epidemiological evidence for a relationship between microbial environment and age-adjusted disease burden. Evolution, Medicine, and Public Health, (1), 173186.

- García Ballester, L. y Mc Vaugh M.R. (eds.) (1996). Arnaldi de Villanova. Opera medica Omnia, vol. X.1. Régimen sanitatis ad regem Aragonum. Barcelona: Autor.

- Gerez Alum, P. (2008). Higiene e imagen de higiene en la baja Edad Media; el caso de la ciudad de Gerona. (Tesis Doctoral). Universidad Nacional de Educación a Distancia, Facultad de Geografía e Historia, España

- Grice, E.A. y Segre, J.A. (2011). The skin microbiome. Nature Reviews Microbiology, 9(4), 244-253.

- Hand, M., Shove, E. y Southerton, D. (2005). Explaining Showering: a discussion of the material, conventional, and temporal dimensions of practice. Sociological Research Online, 10(2). doi:10.5153/sro.1100

- Jumaa, P.A. (2005). Hand hygiene: simple and complex. International Journal of Infectious Diseases, 9, 3-14.

- Kramer, A., Bekeschus, S., Bröker, B.M., Schleibinger, H., Razavi, B. y Assadian, O. (2013). Maintaining health by balancing microbial exposure and prevention of infection: the hygiene hypothesis versus the hypothesis of early immune challenge. Journal of Hospital Infection, 83, S29-S34.

- Lederberg, J. (2001). “Ome sweet” omics-A genealogi- 
cal treasury of words. The Scientist, 15(7).

- Logan, A.C., Katzman, M.A. y Balanzá-Martínez, V. (2015). Natural environments, ancestral diets, and microbiol ecology: is there a modern "paleo-deficit disorder"? part II. Journal of Psysiological Anthropology, 34(9), 1-21.

- Márquez, J., Casas, A. y Estrada, V. (Eds.) (2004). Higienizar, medicar, gobernar. Historia, medicina y sociedad en Colombia. Medellín: Lealon.

- Moreno, P. L. y Viñao, A. (2000). Introducción. Areas. Revista de Ciencias sociales, 20, 7.

- Pehlivan M, Kürtüncü M, Tüzün E, Shugaiv E, Mutlu M, Eraksoy M. y Akman-Demir, G. (2011). The comparison of socio-economic conditions and personal hygiene habits of neuro-BehÇet's disease and multiple sclerosis patients. International Journal of Hygiene and Environmental Health, 214, 335-337.

- Piédrola Gil, G. (2002). Medicina preventiva y salud pública (10 a ed.). Barcelona: Masson.
- Real Academia Española. (2014). Diccionario de la lengua española (23 a ed.). Madrid: Autor.

- Sánchez González, M.A. (2002). Historia, teoría y método de la medicina: introducción al pensamiento médico. Barcelona: Masson.

- Vega Angarita, O.M. y González Escobar, D.S. (2007) Teoría del déficit de autocuidado: interpretación desde los elementos conceptuales. Ciencia y cuidado, 4(4), 28-35.

- Vigarello, G. (1991). Lo limpio y lo sucio. La higiene del cuerpo desde la Edad Media. Madrid: Alianza.

- Vigarello, G. (2006). Lo sano y lo malsano. Historia de las prácticas de la salud desde la Edad Media hasta nuestros dias. Madrid: Abada Editores.

- Volcy, C. (2004). Lo malo y lo feo de los microbios. Bogotá: Universidad Nacional de Colombia.

- Whittemore, R. y Knafl, K. (2005). The integrative review: updated methodology. Journal of Advanced Nursing, 52(5), 546-553. 